\section{An audit on outpatient hysteroscopy - cervical dilatation, failure rates and patient satisfaction}

\author{
Sophie Boyd* and S Lalchandani
}

Department of Obstetrics and Gynaecology, University Hospital Kerry, Ireland

\section{Abstract}

Aims: To audit the use of cervical dilators, local anaesthetic, and failure rates in outpatient hysteroscopy over a two-year period in University Hospital Kerry. To review the experiences of women attending the outpatient hysteroscopy clinic $(\mathrm{OHC})$ over a two-year period in University Hospital Kerry.

Methods: Retrospective data review was carried out. Green-top Guideline No. 59: Best Practice in Outpatient Hysteroscopy, published by the Royal College of Obstetricians and Gynaecologists (RCOG), was the standard used for comparison.

Results: Two hundred and twenty women were seen over a two-year period. The average age was forty-eight. The most common complaint being of menorrhagia/irregular bleeding per vaginum (PV).

Local anaesthetic was used in just under one third of cases of which half required cervical dilatation. Most women reported experiencing mild to moderate levels of discomfort however most would opt for an outpatient hysteroscopic procedure again if required.

Discussion/Conclusion: Outpatient hysteroscopy is a well-tolerated and safe procedure. Suitability for outpatient hysteroscopy is not predictable based on parity of menopausal status. Women would elect to undergo outpatient hysteroscopy again if required and this is likely due to several reasons including convenience and lack of requirement for general anaesthetic.

\author{
More Information \\ *Address for Correspondence: Sophie Boyd, \\ Department of Obstetrics and Gynaecology, \\ University Hospital Kerry, Ireland, \\ Tel: +353 867907646 ; \\ Email: saboyd@tcd.ie; sophie.boyd@hse.ie \\ Submitted: December 02, 2020 \\ Approved: January 11, 2021 \\ Published: January 12, 2021
}

How to cite this article: Boyd S, Lalchandani S. An audit on outpatient hysteroscopy - cervical dilatation, failure rates and patient satisfaction. Clin J Obstet Gynecol. 2021; 4: 003-006.

DOI: 10.29328/journal.cjog.1001076

ORCiD: orcid.org/0000-0001-5623-5958

Copyright: (c) 2021 Boyd S, et al. This is an open access article distributed under the Creative Commons Attribution License, which permits unrestricted use, distribution, and reproduction in any medium, provided the original work is properly cited.

Check for updates

OPEN ACCESS

\section{Introduction}

Outpatient hysteroscopy is an established and widely used diagnostic test. It is carried out primarily to investigate abnormal uterine bleeding [1]. Outpatient hysteroscopy whether diagnostic or operative, is considered a successful, safe, and well tolerated procedure that negates the requirement for general or regional anaesthesia [2]. Hysteroscopic equipment is used to directly visualise and examine the uterine cavity. Advances in the area have allowed operative hysteroscopic procedures such as endometrial polypectomy, submucosal fibroid resection, retrieval of intrauterine contraceptive devices (IUCDs) and directed biopsies to be performed in the outpatient setting with or without the use of local anaesthesia [3]. Benefits of an outpatient hysteroscopy service include no requirement for formal theatre facilities or general/regional anaesthesia, short periods of down time between patients, no requirement for day case/inpatient beds and reduced wait lists and overall cost [4]. Disadvantages include pain associated with instrumentation of the uterus and feeling anxious or embarrassed. The Royal College of Physicians Ireland (RCPI) recommended that a 'one stop shop' should be the standard of care for our patients [3]. This incorporates taking a history, clinical examination and hysteroscopic investigation all being done in one visit with a follow up plan, where indicated, being put in place depending on the clinical findings.

The outpatient hysteroscopy clinic (OHC) at University Hospital Kerry is a Consultant lead service in a dedicated outpatient hysteroscopy suite 'Cill Ide'. Equipment includes 30 degree, $3 \mathrm{~mm}$ diagnostic hysteroscopes and operative alphascopes. A bipolar Gynecare Versapoint Bipolar Electrosurgery system is also used for operative procedures including treatment of endometrial polyps and small submucous leiomyomas. Intracervical Mepivacaine is used for selected cases. To ensure standard practice by all clinicians a dedicated outpatient hysteroscopy proforma is used. In advance of procedure women are sent an information leaflet 
addressing common questions. During the procedure 'vocal local', i.e., conversing with the woman, is used to reduce anxiety and involves women in their procedure. Following the procedure women are seen by the performing doctor and given a follow up care information leaflet which also contained frequently asked questions as well as contact details for follow up concerns and queries. Following the consultation, a patient satisfaction survey is performed to aid ongoing audit and identify areas for improvement.

The overall aims were to firstly audit the use of cervical dilators, local anaesthetic, and failure rates in outpatient hysteroscopy. This was particularly important to assess whether inclusion or exclusion criteria should be used when accepting referrals to minimise failure rates and to reduce delays in patients receiving appropriate care. The second aim was to review the experiences of women attending the outpatient hysteroscopy clinic and to identify areas for improvement.

\section{Methods}

Data was collected at the time of clinic attendance and was stored on a secure hospital computer in a Microsoft Excel spreadsheet. All data was kept anonymised and verbal consent obtained to record and retain information. A retrospective audit was carried out on data collected over a two-year period in one Consultant led clinic from $1^{\text {st }}$ January 2017 to $31^{\text {st }}$ December 2018. All patients who attended the outpatient hysteroscopy clinic over this period and underwent a procedure were included. There were no exclusion criteria applied to these women.

The data was analyzed by the chief author and reviewed by the supervising consultant, second author. Data was analysed using Microsoft Excel.

The RCOG Green-top Guideline No. 59, 'Best practice in outpatient hysteroscopy' [2] was used as the standard for auditing the service.

Data collected included basic demographics such as age as well as obstetric and gynaecological history i.e., parity and menopausal status, relevant medical and surgical history, risk factors including history of cervical procedures, multiple caesarean sections, nulliparity, vaginismus etc. Data collected regarding the procedure included indication, use of dilators or local anaesthetic and if any operative procedures were performed or complications encountered.

Data collected did not include any personal identifying information.

The patient satisfaction survey was made up of four questions. The survey was carried out after the procedure was complete and after the patient had had a consultation regarding findings and follow up plans. The questions asked are listed.
1. If they had read the information sheet sent out prior to the appointment.

2. To give a pain score from $0-10$ using a visual analogue scale (VAS), seen below.

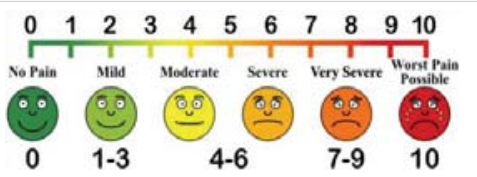

3. To rate their overall experience on a five-point scale from poor to excellent.

4. Whether they would attend the outpatient hysteroscopy service again.

\section{Results}

One weekly led consultant clinic was subject to audit over a two-year period from January 2017 to December 2018. Two hundred and twenty women attended for hysteroscopy and four women attended for insertion of IUCD only.

Most women $(n=105)$ were aged in their forties (Figure 1$)$. Twenty threepercent $(n=51)$ of women were postmenopausal. Eighty eight percent $(n=176)$ of women were multiparous with 48 of those women having exclusively had caesarean section deliveries. Indication for hysteroscopy can be seen in the chart (Figure 2) with $43 \%$ ( $n=95)$ of women presenting with menorrhagia +/- irregular bleeding PV. A small number of women attended for hysteroscopy following incidental findings on pelvic ultrasonography.

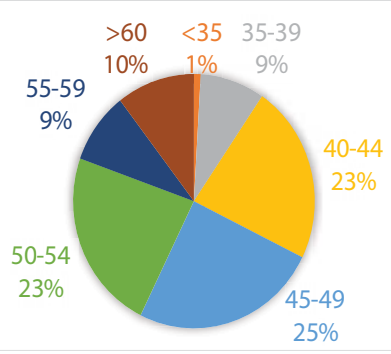

Figure 1: Age, in years, of women attending $\mathrm{OHC}$.

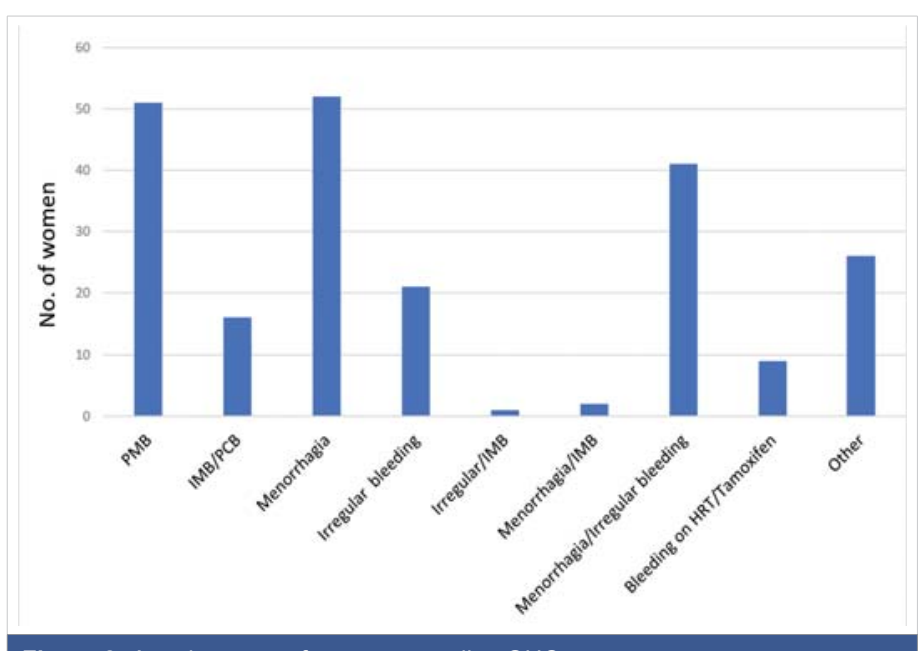

Figure 2: Age, in years, of women attending OHC. 
Sixteen percent $(n=33)$ of women underwent cervical dilation with Hegar cervical dilators. The flow chart below demonstrates the demographics of these women.

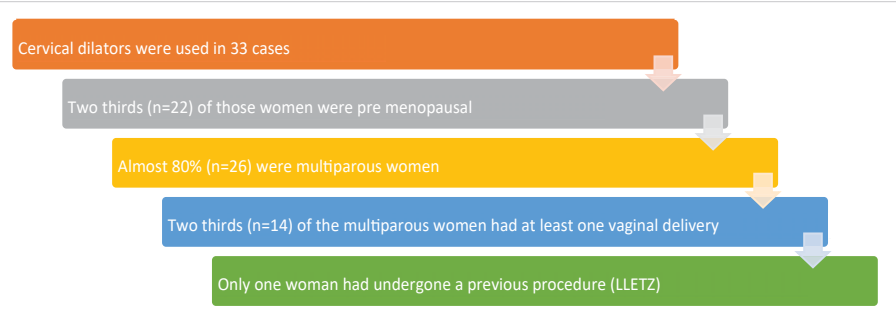

Intracervical Mepivacaine 3\% was used in 70 cases (31\%). All women who underwent cervical dilation received local anaesthetic. An operative procedure was completed on nine women successfully.

On the day of procedure $3 \%,(n=7)$ were cancelled. Reasons included request for procedure under general anaesthetic $(n=3)$, current menses $(n=2)$, prior hysteroscopy within six months $(n=1)$ and large cervical fibroid $(n=1)$. Nineteen procedures were abandoned, $84 \%(n=16)$ were multiparous women, reasons for abandoning procedure are seen in the bar diagram (Figure 3).

Over the same two-year period two hundred and twenty women completed the patient satisfaction survey. Ninetyseven percent $(n=213)$ reported reading the information leaflet they had received in the post with their appointment.

Most women reported the procedure to be mild to moderate in level of discomfort. The graph (Figure 4) demonstrates the
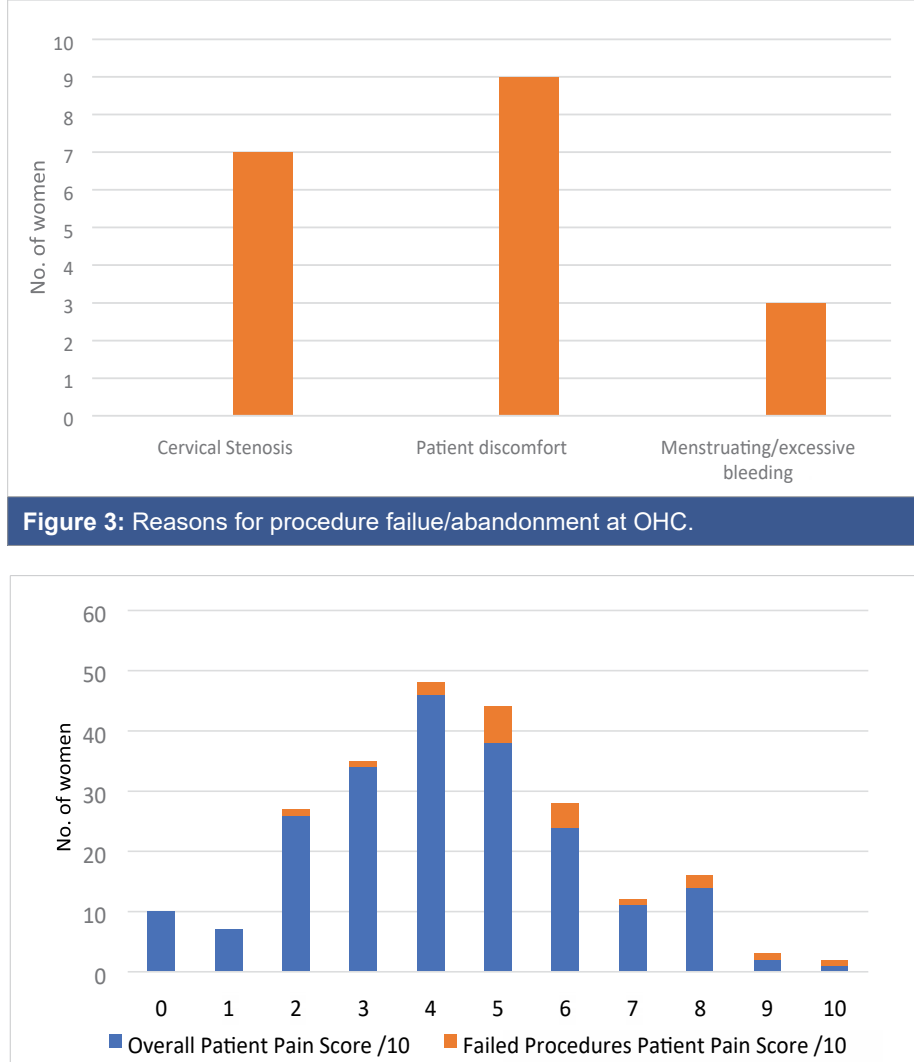

Figure 4: Reasons for procedure failure/abandonment at $\mathrm{OHC}$. pain scores and highlights the women of whom had a failed hysteroscopic procedure.

Women were asked to rate their overall experience in the outpatient hysteroscopy suite. They were asked to give a rating of either poor, average, good, very good or excellent. As demonstrated (Figure 5) many women had a good $(n=127)$, very good $(n=36)$ or excellent $(n=19)$ experience.

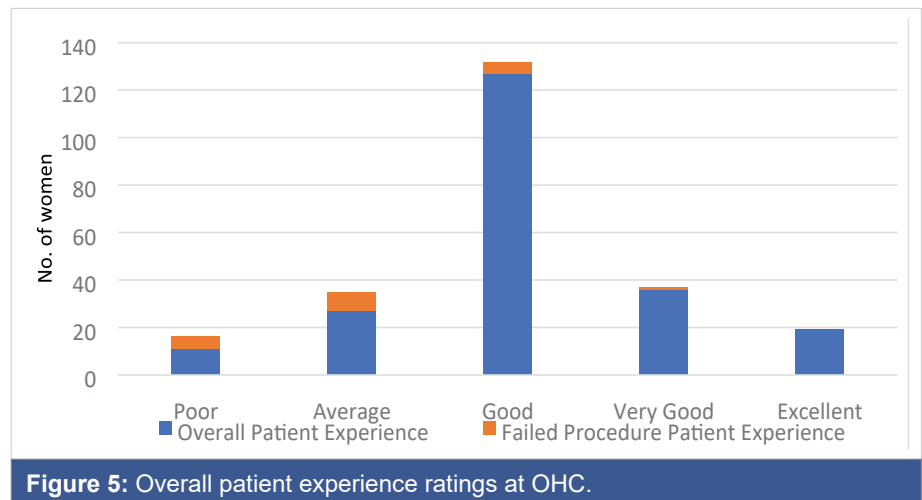

A small number of women had a poor $(n=11)$ or average ( $n=27$ ) experience. The women for whom a procedure was not completed are highlighted on the bar chart.

The final question of the patient satisfaction survey was whether a woman would choose to return if there was an indication to do so in the future. Ninety percent of women ( $n=198)$ reported that they would return to the outpatient hysteroscopy suite if required.

\section{Discussion}

Outpatient diagnostic hysteroscopy is an accurate and reliable way to assess for intrauterine abnormalities [5]. The audit demonstrated that over $91 \%$ of hysteroscopic procedures were successfully completed, meeting the 'one stop shop' standard of care [3]. Cervical dilators and local anaesthetic were used in a number subset of cases; $16 \%$ and $31 \%$ respectively, without complications. Use of local anaesthetic does not necessarily help reduce pain but can reduce the incidence of vasovagal reactions during cervical dilation [2]. It is recommended to use topical anaesthetic if using a tenaculum to grasp the cervix [2]. The use of local anaesthetic likely increases tolerance for the procedure and thus patient satisfaction.

Overall outpatient hysteroscopy is a well-tolerated procedure. Despite a small number of women experiencing moderate to severe pain, the majority would still elect to have an outpatient hysteroscopy versus alternative options i.e., hysteroscopy under general anaesthesia. The reason for this is likely multifactorial, undergoing an immediate procedure may reduce pre-procedure anxiety particularly due to not anticipating a regional or general anaesthetic [8]. Average pain score was 4/10 (moderate) and the overall average experience was good. There was no significant 
difference in pain experienced by post- menopausal women despite an expected difference in vaginal and cervical atrophy [7]. The results demonstrate that suitability for outpatient hysteroscopy is not predictable based on multiparity or menopausal status and thus all women should be offered the option of an ambulatory procedure [6].

Most women would attend the outpatient hysteroscopy unit again if required in the future. The convenience of not significantly disrupting daily routines is likely a contributing factor [8]. The presence of nursing support staff and direct observation of the hysteroscopy camera view on a screen within the room likely also aids tolerance of the procedure by partially distracting from discomfort [8]. 'Vocal local' reduces anxiety and the involvement in the procedure also likely improves satisfaction.

The eight percent of women who experienced an abandoned procedure were more likely to have had a 'poor' or 'average' overall experience. This is likely due to an increase in amount of pain experience and the anticipation of having to undergo another procedure with regional or general anaesthesia.

Consideration should be made for the appropriate use of timed hormonal medication to reduce the number of cancelled or abandoned procedures due to menstrual bleeding.

Recommending the use of pre procedure simple analgesia may reduce patient discomfort and subsequent abandonment of procedure. Reduced discomfort is likely to improve overall experience and thus see higher patient numbers electing to have outpatient hysteroscopy reducing inpatient patient waiting lists and overall hospital cost.

There is a possible role of pre procedural misoprostol in women with factors that may contribute to cervical stenosis i.e., postmenopausal, nulliparous, multiple caesarean sections, LLETZ (large loop excision of transformation zone) procedures etc. Again, this may further reduce discomfort and failure rates.
In conclusion the outpatient hysteroscopy clinic has a high rate of successfully completing procedures and a high level of patient satisfaction. Several areas were identified to improve patient experience and satisfaction including pre procedure analgesia recommendations and timed hormonal treatment to reduce cancellations as well as patient comfort.

\section{References}

1. Clark TJ, Voit D, Gupta JK, Hyde C, Song F, et al. Accuracy of Hysteroscopy in the Diagnosis of Endometrial Cancer and Hyperplasia - A Systematic Quantitative Review. JAMA. 2002; 288: 1610-1621. PubMed: https://pubmed.ncbi.nlm.nih.gov/12350192/

2. Royal College of Obstetricians and Gynaecologists. Green-top. Best Practice in Outpatient Hysteroscopy. Guideline No. 59. 2011.

3. Clinical Practice Guideline: The Investigation and Management of Menorrhagia. Institute of Obstetricians and Gynaecologists, Royal College of Physicians of Ireland. Guideline No: 7. 2015.

4. Kremer C, Duffy S, Moroney M. Patient satisfaction with outpatient hysteroscopy versus day case hysteroscopy: randomised controlled trial. BMJ. 2000; 320: 279-282.

PubMed: https://pubmed.ncbi.nlm.nih.gov/10650023/

5. van Dongen $\mathrm{H}$, de Kroon $\mathrm{CD}$, Jacobi $\mathrm{CE}$, Trimbos JB, Jansen FW. Diagnostic hysteroscopy in abnormal uterine bleeding: a systematic review and meta-analysis. BJOG. 2007; 114: 664-675. PubMed: https://pubmed.ncbi.nlm.nih.gov/17516956/

6. Litta P, Cosmi E, Saccardi C, Esposito C, Rui R, et al. Outpatient operative polypectomy using a $5 \mathrm{~mm}$-hysteroscope without anaesthesia and/or analgesia: advantages and limits. Eur J Obstet Gynecol Reprod Biol. 2008; 139: 210-214.

PubMed: https://pubmed.ncbi.nlm.nih.gov/18248873/

7. Downes E, Al-Azzawi F. How well do perimenopausal patients accept outpatient hysteroscopy? Visual analogue scoring of acceptability and pain in 100 women. Eur J Obstet Gynecol Reprod Biol. 1993; 48: 37-41. PubMed: https://pubmed.ncbi.nlm.nih.gov/8449260/

8. Morgan M, Dodds W, Wolfe C, Raju S. Women's views and experiences of outpatient hysteroscopy: implications for a patient-centered service. Nurs Health Sci. 2004; 6: 315-320.

PubMed: https://pubmed.ncbi.nlm.nih.gov/15507052/ 